\title{
Poorly controlled gout: who is doing poorly?
}

Faith Li-Ann Chia, MBBS, FRCP

\begin{abstract}
Gout, an inflammatory arthritis caused by the deposition of monosodium urate crystals, is commonly seen in primary care and specialist clinics. In recent years, there has been a resurgence of interest in gout due to advances in therapies and the understanding of pathophysiology, with new guidelines being published by international bodies. However, there is still a gap between the goals of treatment and actual day-to-day practice. Barriers that result in poorly controlled gout include patient factors such as lack of understanding of the disease, stigma and nonadherence to treatment, as well as physician factors such as knowledge gaps, inadequate use of allopurinol and lack of ownership of the disease. The medical profession needs to do more to bridge the gap through physician and patient education, identification of treatment targets with appropriate use of drugs, and dissemination of guidelines.
\end{abstract}

Keywords: allopurinol, drug hypersensitivity, hyperuricaemia, medication adherence

\section{INTRODUCTION}

Gout is a common condition caused by deposition of monosodium urate (MSU) crystals that occurs after chronic elevation of uric acid levels above its saturation point of $360 \mu \mathrm{mol} / \mathrm{L}(6 \mathrm{mg} / \mathrm{dL}){ }^{(1)}$ It affects an estimated 6.1 million adults in the United States and its incidence in Asia is rising to match these figures. ${ }^{(2,3)}$ Men are more often affected, but the disparity between genders decreases with increasing age due to declining levels of oestrogen, which possesses uricosuric effects, in older women. ${ }^{(4)}$ There is a strong association between gout and other disorders of the metabolic syndrome, which are on the rise in Singapore. ${ }^{(5)}$ Hyperuricaemia not only causes painful attacks of gout and joint damage, but is increasingly reported as an independent risk factor for cardiovascular disease and mortality. ${ }^{(6)}$ In the last few years, there has been a resurgence of interest in gout due to the approval of new drugs such as febuxostat and an improved understanding of the inflammasome pathway that mediates the inflammatory response in gout. $^{(7)}$

\section{WHAT SHOULD WE BE ACHIEVING?}

The European League Against Rheumatism (EULAR) has published a set of evidence-based guidelines for gout management, emphasising that the optimal treatment of gout should include non-pharmacological modalities and patient education. It highlighted that the therapeutic goal of urate-lowering therapy was to promote crystal dissolution and prevent crystal formation by maintaining the serum uric acid (SUA) level below the saturation point for MSU $(<360 \mu \mathrm{mol} / \mathrm{L}){ }^{(8)}$ The British Society for Rheumatology and American College of Rheumatology (ACR) published similar recommendations. ${ }^{(9,10)}$ Tophus size was also demonstrated to reduce more rapidly in patients with lower SUA levels. ${ }^{(11)}$

\section{HOW WELL ARE WE DOING?}

Although we understand the pathophysiology of gout and have a drug armamentarium available to treat it, the management of gout remains poor. Studies in the primary care setting in the United Kingdom showed that $20 \%$ of patients with tophaceous gout and $33 \%$ of patients with recurrent attacks were on allopurinol, and only $33 \%$ of patients on urate-lowering therapy attained the SUA treatment target. ${ }^{(12,13)}$ Similar results were seen locally: an audit of the rheumatology clinic in a restructured hospital found that only $25 \%$ of patients started on allopurinol had achieved their target SUA levels at one-year follow-up. ${ }^{(3)}$

\section{BARRIERS TO THE OPTIMAL TREATMENT OF GOUT}

Several factors contribute to the gap between the optimal treatment of gout and the reality of daily practice.

\section{Patient factors}

One of the most important patient factors is the lack of understanding that gout is a chronic disease. Patients may brush off attacks as being inconvenient with no effect on their health, seek pain relief and believe that diet control is the only treatment option. Some patients and health professionals think that gout is a disease that reflects poor self-control, an unhealthy lifestyle, gluttony and excessive alcohol consumption. This may lead to embarrassment and a reluctance to seek treatment on the part of the patient. Furthermore, the predominantly male patients may feel that they have to appear 'manly' and bear with the symptoms. ${ }^{(14)}$

Adherence rates to pharmacotherapy in gout are particularly poor, ranging from $18 \%-44 \%$, and significantly lower than those in patients with other chronic diseases. ${ }^{(15,16)}$ Reasons offered by patients who were nonadherent include the belief that they were cured, fear of side effects and pill burden;(14) this is especially relevant in the local context as most formulations of allopurinol are only available in 100-mg tablets.

\section{Physician factors}

The belief that gout is not a chronic disease is also a barrier among physicians. Studies among health professionals have shown 
that many perceive gout as an acute disease and presume that patients would prefer to take painkillers when necessary rather than be on long-term medication. ${ }^{(14)}$ In a healthcare system such as Singapore's where patients tend to visit a variety of doctors and hospitals, there may be inertia to start a new drug unless the patient sees the same doctor who can appreciate their recurrent attacks.

Many physicians are not aware of guidelines for gout. This is unsurprising since most guidelines are published in rheumatology journals and may not reach the primary care setting, where a majority of patients are treated. ${ }^{(17)}$ Knowledge of the target SUA that patients need to achieve is limited - a study that surveyed attendees of a major rheumatology conference showed that most thought the target of treatment was reduction in attacks, with only $27 \%$ of respondents correctly answering that the optimum SUA level was less than $360 \mu \mathrm{mol} / \mathrm{L} .{ }^{(18)}$

Urate-lowering therapy is a crucial component for adequate control of gout. Indications for commencement of urate-lowering therapy include recurrent attacks, presence of tophi, joint damage or nephrolithiasis. ${ }^{(8)}$ Allopurinol remains the primary option for long-term urate-lowering therapy, but is frequently underused and underdosed. One factor that may limit the use of allopurinol is concerns about side effects and severe hypersensitivity reactions. A minority of patients may develop serious drug reactions such as drug rash with eosinophilia and systemic symptoms (DRESS) or severe cutaneous adverse reactions (SCAR), including StevensJohnson syndrome and toxic epidermal necrolysis. ${ }^{(1)}$ In a local study on hospitalised patients with drug allergies, allopurinol was the culprit drug in $5.7 \%$ of patients and accounted for $38 \%$ of cases of DRESS; it was one of the top three causative drugs in SCAR. ${ }^{(19)}$ Patient deaths due to allopurinol allergy have been featured in local newspapers over the past decade and resulted in two 'Dear Healthcare Professional Letters' issued by the Health Sciences Authority, Singapore, which may have increased the fear of using allopurinol. ${ }^{(20,21)}$

The maximum dose of allopurinol is 800 mg daily; however, it is often administered in a fixed dose of $300 \mathrm{mg}$. This dose is insufficient for most patients to achieve their target SUA level. An observational study in the United Kingdom showed that the median dose of allopurinol required to reach a target SUA level was $400 \mathrm{mg}$ daily, ${ }^{(22)}$ while in randomised trials in the United States, a dose of $300 \mathrm{mg}$ daily only achieved targets in $20 \%$ of patients. ${ }^{(23)}$ An initial dose of $100 \mathrm{mg}$ daily is recommended, with gradual increments of 100 mg every $2-5$ weeks until target SUA levels are achieved. Allopurinol can be initiated during attacks as long as anti-inflammatory therapy is administered at the same time. ${ }^{(10)}$

Hande et al's algorithm for the dosing of allopurinol based on creatinine clearance is deeply ingrained in many physicians' practices. ${ }^{(24)}$ However, limiting the dose of allopurinol results in suboptimal control of gout, particularly since many patients with gout have renal impairment. ${ }^{(25)}$ Studies show that administering an allopurinol dosage that is above the 'cap' does not increase toxicity in patients with renal impairment and results in a significant reduction in SUA levels. ${ }^{(26,27)}$ Furthermore, a recent study has shown that in patients with chronic renal disease and hyperuricaemia, the use of urate-lowering therapy may slow the progression of renal disease. ${ }^{(28)}$ The EULAR and ACR guidelines recommend starting with allopurinol $50 \mathrm{mg}$ once daily in patients who have Stage 4 chronic kidney disease or worse and gradually titrating the dose by 50-mg increments until target SUA levels are reached, while monitoring for toxicity.

Another important aspect of physician education that needs improvement is the use of prophylaxis against gout flares during initiation of urate-lowering therapy. These attacks can be very discouraging and may cause patients to abandon compliance to treatment. Slow titration of urate-lowering therapy and the use of prophylactic agents can decrease the risk of flares. ${ }^{(29)}$ The two first-line options for prophylaxis are low-dose colchicine $(0.5 \mathrm{mg}$ daily or twice daily) and low-dose nonsteroidal anti-inflammatory drugs, which can be used for up to six months or until target SUA levels are reached. ${ }^{(30)}$

Finally, there may be a perceived lack of options for uratelowering therapy in patients who have adverse drug reactions to allopurinol. Patients with simple rash due to allopurinol can undergo allopurinol desensitisation using a well-established protocol. ${ }^{(31)}$ Febuxostat is a non-purine xanthine oxidase inhibitor that has been shown to be effective in decreasing SUA levels without dose adjustment or concerns over toxicity in patients with renal impairment. ${ }^{(23,32)}$ It is not presently registered for use in Singapore, but is available as an exemption drug. Uricosuric agents such as probenecid and benzbromarone can be used in patients who fail to reach their targets on allopurinol or cannot tolerate it. The combination of drugs with different modes of action has also been shown to improve urate-lowering therapy. ${ }^{(7,33)}$ Other drugs such as losartan, fenofibrate and oral vitamin C supplements also have direct uricosuric effects and may be considered for adjunctive treatment. ${ }^{(4)}$

\section{HOW DO WE BRIDGE THE GAP?}

Optimal treatment of gout is achievable in routine clinical practice with changes in physician prescribing habits, a multidisciplinary approach, and increased patient education and empowerment. A local study showed that implementing process changes increased the percentage of gout patients achieving target SUA levels from $27 \%$ to $72 \%$ at one year. . $^{(3)}$ Another study showed that education changed adherence dramatically, with 92\% of patients achieving their target after a predominantly nurse-led intervention involving counselling. ${ }^{(22)}$

It is clearly important that we improve the current treatments for gout. Transferring the care of gout to the tertiary level is not the answer to the problem, as rheumatologists have been shown to be no better than primary care physicians at treating gout. Changing medical practices may not be easy, but steps that we should take include greater public awareness of gout as a chronic disease, improved education among the medical community and dissemination of guidelines for gout. An inter-professional approach involving physicians, nurses, clinical pharmacists and dieticians is essential, and patients should receive both verbal and written information on gout. We should also define the 
targets of treatment - both in terms of SUA levels and quality indicators. Patients who have multiple comorbidities, cannot tolerate allopurinol or are allergic to allopurinol and require desensitisation should be referred to a specialist. ${ }^{(17)}$ Hopefully, by taking these steps and using allopurinol appropriately with SUA targets, we can deliver the best care to patients with gout.

\section{ACKNOWLEDGEMENT}

I would like to thank the late Professor Feng Pao Hsii for his guidance with this manuscript.

\section{REFERENCES}

1. Rees F, Hui M, Doherty M. Optimizing current treatment of gout. Nat Rev Rheumatol 2014; 10:271-83.

2. Lawrence RC, Felson DT, Helmick CG, et al; National Arthritis Data Workgroup. Estimates of the prevalence of arthritis and other rheumatic conditions in the United States. Part II. Arthritis Rheum 2008; 58:26-35.

3. Lim AY, Shen L, Tan $\mathrm{CH}$, et al. Achieving treat to target in gout: a clinical practice improvement project. Scand J Rheumatol 2012; 41:450-7.

4. Neogi T. Clinical practice. Gout. N Engl J Med 2011; 364:443-52.

5. Zhu Y, Pandya BJ, Choi HK. Comorbidities of gout and hyperuricemia in the US general population: NHANES 2007-2008. Am J Med 2012; 125:679-687.e1.

6. Stack AG, Hanley A, Casserly LF, et al. Independent and conjoint associations of gout and hyperuricaemia with total and cardiovascular mortality. QJM 2013; 106:647-58.

7. Jansen TL. Treat to target in gout by combining two modes of action. Rheumatology (Oxford) 2014; 53:2131-3.

8. Zhang W, Doherty M, Bardin T, et al; EULAR Standing Committee for International Clinical Studies Including Therapeutics. EULAR evidence based recommendations for gout. Part II: Management. Report of a task force of the EULAR Standing Committee for International Clinical Studies Including Therapeutics (ESCISIT). Ann Rheum Dis 2006; 65:1312-24.

9. Jordan KM, Cameron JS, Snaith M, et al; British Society for Rheumatology and British Health Professionals in Rheumatology Standards, Guidelines and Audit Working Group (SGAWG). British Society for Rheumatology and British Health Professionals in Rheumatology guideline for the management of gout. Rheumatology (Oxford) 2007; 46:1372-4.

10. Khanna D, Fitzgerald JD, Khanna PP, et al; American College of Rheumatology. 2012 American College of Rheumatology guidelines for management of gout. Part 1: systematic nonpharmacologic and pharmacologic therapeutic approaches to hyperuricemia. Arthritis Care Res (Hoboken) 2012; 64:1431-46.

11. Perez-Ruiz F, Calabozo M, Pijoan JI, Herrero-Beites AM, Ruibal A. Effect of urate-lowering therapy on the velocity of size reduction of tophi in chronic gout. Arthritis Rheum 2002; 47:356-60.

12. Roddy E, Zhang W, Doherty M. Concordance of the management of chronic gout in a UK primary-care population with the EULAR gout recommendations. Ann Rheum Dis 2007; 66:1311-5.

13. Cottrell E, Crabtree V, Edwards JJ, Roddy E. Improvement in the management of gout is vital and overdue: an audit from a UK primary care medical practice. BMC Fam Pract 2013; 14:170.

14. Spencer K, Carr A, Doherty M. Patient and provider barriers to effective management of gout in general practice: a qualitative study. Ann Rheum Dis 2012; 71:1490-5.
15. Reach G. Treatment adherence in patients with gout. Joint Bone Spine $2011 ; 78: 456-9$

16. Harrold LR, Andrade SE, Briesacher BA, et al. Adherence with uratelowering therapies for the treatment of gout. Arthritis Res Ther 2009; $11:$ R46.

17. Perry ME, Madhok R. Treatment failure gout: failure to treat? Rheumatology (Oxford) 2010; 49:2233-4.

18. Doherty M, Bardin T, Pascual E. International survey on the diagnosis and management of gout. Ann Rheum Dis 2007; 66:1685-6.

19. Thong BY, Leong KP, Tang CY, Chng HH. Drug allergy in a general hospital: Results of a novel prospective inpatient reporting system. Ann Allergy Asthma Immunol 2003; 90:342-7.

20. Health Sciences Authority, Singapore. Risk assessment of allopurinol. Available at: http://www.hsa.gov.sg/content/hsa/en/Health_Products_ Regulation/Safety_Information_and_Product_Recalls/Dear_Healthcare_ Professional_Letters/2001/Risk_assessment_of_allopurinolbr_.html. Accessed July 19, 2016.

21. Health Sciences Authority, Singapore. Serious skin reactions associated with allopurinol. In: Adverse Drug Reaction News [online]. Available at: http://www.hsa.gov.sg/content/dam/HSA/HPRG/Safety_Alerts_Product_ Recalls_Enforcement/Adverse_Drug_Reaction_News/2009/ADR_News_ Aug2009_Vol11_No2.pdf. Accessed July 19, 2016.

22. Rees F, Jenkins W, Doherty M. Patients with gout adhere to curative treatment if informed appropriately: proof-of-concept observational study. Ann Rheum Dis 2013; 72:826-30.

23. Becker MA, Schumacher HR Jr, Wortmann RL, et al. Febuxostat compared with allopurinol in patients with hyperuricemia and gout. N Engl J Med 2005; 353:2450-61.

24. Hande KR, Noone RM, Stone WJ. Severe allopurinol toxicity. Description and guidelines for prevention in patients with renal insufficiency. Am J Med 1984; 76:47-56.

25. Dalbeth N, Kumar S, Stamp L, Gow P. Dose adjustment of allopurinol according to creatinine clearance does not provide adequate control of hyperuricemia in patients with gout. J Rheumatol 2006; 33:1646-50.

26. Vázquez-Mellado J, Morales EM, Pacheco-Tena C, Burgos-Vargas R. Relation between adverse events associated with allopurinol and renal function in patients with gout. Ann Rheum Dis 2001; 60:981-3.

27. Stamp LK, O'Donnell JL, Zhang M, et al. Using allopurinol above the dose based on creatinine clearance is effective and safe in patients with chronic gout, including those with renal impairment. Arthritis Rheum $2011 ; 63: 412-21$

28. Levy GD, Rashid N, Niu F, Cheetham TC. Effect of urate-lowering therapies on renal disease progression in patients with hyperuricemia. J Rheumatol 2014; 41:955-62.

29. Borstad GC, Bryant LR, Abel MP, et al. Colchicine for prophylaxis of acute flares when initiating allopurinol for chronic gouty arthritis. J Rheumatol 2004; 31:2429-32

30. Latourte A, Bardin T, Richette P. Prophylaxis for acute gout flares after initiation of urate-lowering therapy. Rheumatology (Oxford) 2014; 53:1920-6.

31. Fam AG, Lewtas J, Stein J, Paton TW. Desensitization to allopurinol in patients with gout and cutaneous reactions. Am J Med 1992; 93:299-302.

32. Schumacher HR Jr, Becker MA, Wortmann RL, et al. Effects of febuxostat versus allopurinol and placebo in reducing serum urate in subjects with hyperuricemia and gout: a 28-week, phase III, randomized, double-blind, parallel-group trial. Arthritis Rheum 2008; 59:1540-8.

33. Azevedo VF, Buiar PG, Giovanella LH, Severo CR, Carvalho M. Allopurinol, benzbromarone, or a combination in treating patients with gout: analysis of a series of outpatients. Int J Rheumatol 2014; 2014:263720. 